УДК 519.716

\title{
Classification of Hyperfunctions of Rank 2 with Respect to Membership in the Maximal Partial Ultraclones
}

\author{
Sergey A. Badmaev* \\ Institute of Mathematics and Computer Science \\ Buryat State University \\ Smolina, 24a, Ulan-Ude, 670000 \\ Russia
}

Received 03.04.2019, received in revised form 22.07.2019, accepted 20.09.2019

\begin{abstract}
In this paper, we consider the set of hyperfunctions, which is a subset of the full partial ultraclone of rank 2. For hyperfunctions, the problem of their classification with respect to membership in the the maximal partial ultraclones is solved. The relation of membership in the maximal partial ultraclones is an equivalence relation and generates the corresponding partition into equivalence classes. A complete description of all equivalence classes, the total number of which is 28, is obtained.
\end{abstract}

Keywords: multifunction, hyperfunction, clone, ultraclone, maximal partial ultraclone, classification of functions.

DOI: 10.17516/1997-1397-2019-12-5-645-652.

\section{Introduction}

A sets of multifunctions are considered. A multifunction on a finite set $A$ is a function defined on the set $A$ and taking as its values its subsets. Obviously, superposition in the usual sense does not work when working with multifunctions. Therefore, they need to give a new definition of superposition. Two ways of defining superposition are usually considered: the first is based on the union of subsets of the set $A$, and in this case the closed sets containing all the projections are called multiclones, and the second is the intersection of the subsets of $A$, and the closed sets containing all projections are called partial ultraclones. The set of multifunctions on $A$ on the one hand contains all the functions of $|A|$-valued logic and on the other, is a subset of functions of $2^{|A|}$-valued logic with superposition that preserves these subset.

In the theory of functions the problem of classification is interesting. One of the known variants of the classification of functions of $k$-valued logic is one in which functions in a closed subset $B$ of a closed set $M$ can be divided according to their membership in the classes that are pre-complete in $M$. In this paper, the subset of $B$ is the set of all Boolean functions, and the set of $M$ is the set of all multifunctions on the two-element set, and the partial maximal ultraclones are pre-complete classes.

Note that the quality and type of functions are limited to the function The other k-logic logic is used, for example, in the process [1-8].

*badmaevsa@mail.ru

(C) Siberian Federal University. All rights reserved 


\section{Basic concepts and definitions}

Let $E=\{0,1\}$ и $F=\{\varnothing,\{0\},\{1\},\{0,1\}\}$. We define the following sets of functions:

$P_{2, n}^{\bar{*}}=\left\{f \mid f: E^{n} \rightarrow F\right\}, P_{2}^{\bar{*}}=\bigcup_{n} P_{2, n}^{\bar{*}}$;

$P_{2, n}=\left\{f \mid f \in P_{2, n}^{\bar{*}}\right.$ и $|f(\tilde{\alpha})|=1$ for any $\left.\tilde{\alpha} \in E^{n}\right\}, P_{2}=\bigcup_{n} P_{2, n}$;

$P_{2, n}^{-}=\left\{f \mid f: E^{n} \rightarrow F \backslash\{\varnothing\}\right\}, P_{2}^{-}=\bigcup_{n} P_{2, n}^{-} ;$

$P_{2, n}^{*}=\left\{f \mid f \in P_{2, n}^{\bar{*}}\right.$ and $|f(\tilde{\alpha})| \leqslant 1$ for any $\left.\tilde{\alpha} \in E^{n}\right\}, P_{2}^{*}=\bigcup_{n} P_{2, n}^{*}$.

Functions from $P_{2}$ are called Boolean functions, functions from $P_{2}^{*}$ are called partial functions on $E$, functions from $P_{2}^{-}$are called hyperfunctions on $E$, functions from $P_{2}^{\bar{*}}$ are called multifunctions on $E$.

We believe that the superposition

$$
f\left(f_{1}\left(x_{1}, \ldots, x_{m}\right), \ldots, f_{n}\left(x_{1}, \ldots, x_{m}\right)\right),
$$

where $f, f_{1}, \ldots, f_{n} \in P_{2}^{\bar{*}}$, represents some multifunction $g\left(x_{1}, \ldots, x_{m}\right)$ on a tuple with elements from the set $F$, if for any $\left(\alpha_{1}, \ldots, \alpha_{m}\right) \in E^{m}$

$$
g\left(\alpha_{1}, \ldots, \alpha_{m}\right)= \begin{cases}\bigcap_{\beta_{i} \in f_{i}\left(\alpha_{1}, \ldots, \alpha_{m}\right)} f\left(\beta_{1}, \ldots, \beta_{n}\right) & \text { if the intersection is not empty; } \\ \bigcup_{\beta_{i} \in f_{i}\left(\alpha_{1}, \ldots, \alpha_{m}\right)} f\left(\beta_{1}, \ldots, \beta_{n}\right) & \text { otherwise. }\end{cases}
$$

On tuples containing $\varnothing$, the multifunction takes the value $\varnothing$.

This definition allows you to find the value $f\left(x_{1}, \ldots, x_{n}\right)$ for every $\left(\sigma_{1}, \ldots, \sigma_{n}\right) \in F^{n}$.

For simplicity we use the following code:

$$
\varnothing \leftrightarrow *,\{0\} \leftrightarrow 0,\{1\} \leftrightarrow 1,\{0,1\} \leftrightarrow-.
$$

We note that in this paper we will adhere to the terminology adopted in [9], which will allow us not to introduce additional definitions here.

In [10] it is proved that the maximal partial ultraclones of rank 2 are only the following 12 sets:

1) $K_{1}$ is the set consisting of all multifunctions $f$ such that $f(\tilde{0}) \in\{0, *\}$.

2) $K_{2}$ is the set consisting of all multifunctions $f$ such that $f(\tilde{1}) \in\{1, *\}$.

3) $K_{3}$ is the set consisting of all multifunctions $f$ for which one of the two conditions is fulfilled:

- $f(\tilde{0})=*$ or $f(\tilde{1})=*$,

- $f(\tilde{0})=0$ and $f(\tilde{1})=1$.

4) $K_{4}$ is the set consisting of all multifunctions $f$ such that on any binary tuple $\tilde{\alpha}$ one of three conditions is fulfilled:

- $f(\tilde{\alpha})=f(\overline{\tilde{\alpha}})=-$,

- $f(\tilde{\alpha})=f(\overline{\tilde{\alpha}})=*$,

- $f(\tilde{\alpha})=\overline{f(\overline{\tilde{\alpha}})}$, where $f(\tilde{\alpha}) \in\{0,1\}$. 
5) $K_{5}$ is the set consisting of all multifunctions $f$ such that on any binary tuple $\tilde{\alpha}$ one of two conditions is fulfilled:

- $f(\tilde{\alpha})=*$ or $f(\overline{\tilde{\alpha}})=*$,

- $f(\tilde{\alpha})=\overline{f(\overline{\tilde{\alpha}})}$, where $f(\tilde{\alpha}) \in\{0,1\}$.

6) $K_{6}=P_{2}^{-} \cup\{*\}$.

7) $K_{7}=P_{2}^{*}$

8) $K_{8}$ is the set of all multifunctions $f$ that simultaneously satisfy three conditions:

- if $f(\tilde{\alpha}), f(\tilde{\beta}), f(\tilde{\gamma}) \in\{0,1\}$, then

$$
f\left(\begin{array}{c}
\tilde{\alpha} \\
\tilde{\beta} \\
\tilde{\gamma}
\end{array}\right) \in\left\{\left(\begin{array}{l}
0 \\
0 \\
0
\end{array}\right),\left(\begin{array}{l}
0 \\
0 \\
1
\end{array}\right),\left(\begin{array}{l}
0 \\
1 \\
0
\end{array}\right),\left(\begin{array}{l}
1 \\
1 \\
1
\end{array}\right)\right\},
$$

where $\tilde{\alpha}=\left(\alpha_{1}, \ldots, \alpha_{n}\right), \tilde{\beta}=\left(\beta_{1}, \ldots, \beta_{n}\right), \tilde{\gamma}=\left(\gamma_{1}, \ldots, \gamma_{n}\right)$ are binary tuples such that $\left(\alpha_{i} \beta_{i} \gamma_{i}\right) \in\{(000),(001),(010),(111)\}$ for any $i \in\{1, \ldots, n\}$;

- if there is a binary tuple $\tilde{\alpha}$ such that $f(\tilde{\alpha})=-$, then for any binary tuple $\tilde{\beta}$ true $f(\tilde{\beta}) \neq 1$;

- let binary tuples $\tilde{\alpha}=\left(\alpha_{1}, \ldots, \alpha_{n}\right), \tilde{\beta}=\left(\beta_{1}, \ldots, \beta_{n}\right)$ such that $\alpha_{i} \leqslant \beta_{i}$ for any $i \in\{1, \ldots, n\}$ then, if $f(\tilde{\alpha})=*$, then $f(\tilde{\beta})=*$.

9) $K_{9}$ is the set of all multifunctions $f$ that simultaneously satisfy three conditions:

- if $f(\tilde{\alpha}), f(\tilde{\beta}), f(\tilde{\gamma}) \in\{0,1\}$, then

$$
f\left(\begin{array}{c}
\tilde{\alpha} \\
\tilde{\beta} \\
\tilde{\gamma}
\end{array}\right) \in\left\{\left(\begin{array}{l}
0 \\
0 \\
0
\end{array}\right),\left(\begin{array}{l}
0 \\
1 \\
1
\end{array}\right),\left(\begin{array}{l}
1 \\
0 \\
1
\end{array}\right),\left(\begin{array}{l}
1 \\
1 \\
1
\end{array}\right)\right\}
$$

where $\tilde{\alpha}=\left(\alpha_{1}, \ldots, \alpha_{n}\right), \tilde{\beta}=\left(\beta_{1}, \ldots, \beta_{n}\right), \tilde{\gamma}=\left(\gamma_{1}, \ldots, \gamma_{n}\right)$ are binary tuples such that $\left(\alpha_{i} \beta_{i} \gamma_{i}\right) \in\{(000),(011),(101),(111)\}$ for any $i \in\{1, \ldots, n\}$;

- if there is a binary tuple $\tilde{\alpha}$ such that $f(\tilde{\alpha})=-$, then for any binary tuple $\tilde{\beta}$ true $f(\tilde{\beta}) \neq 0$;

- let binary tuples $\tilde{\alpha}=\left(\alpha_{1}, \ldots, \alpha_{n}\right), \tilde{\beta}=\left(\beta_{1}, \ldots, \beta_{n}\right)$ such that $\alpha_{i} \leqslant \beta_{i}$ for any $i \in\{1, \ldots, n\}$ then, if $f(\tilde{\beta})=*$, then $f(\tilde{\alpha})=*$.

10) $K_{10}$ is the set of all multifunctions preserve the predicate

$$
R_{10}=\left(\begin{array}{llllllllll}
0 & 0 & 0 & 0 & 1 & 1 & 1 & 1 & - & \alpha \\
0 & 0 & 1 & 1 & 1 & 1 & 0 & 0 & - & \beta \\
0 & 1 & 0 & 1 & 1 & 0 & 1 & 0 & - & \gamma \\
0 & 1 & 1 & 0 & 1 & 0 & 0 & 1 & - & \delta
\end{array}\right) \text {, where }(\alpha, \beta, \gamma, \delta)^{t} \text { are all sorts of columns in }
$$

which $\alpha, \beta, \gamma, \delta \in F$ are simultaneously satisfy two conditions:

- in every column $(\alpha, \beta, \gamma, \delta)^{t}$ among $\alpha, \beta, \gamma, \delta$ least two assume the value $*$;

- in every column $(\alpha, \beta, \gamma, \delta)^{t}$, if 0 or 1 are found among $\alpha, \beta, \gamma, \delta$ then all of them are not equal to - . 
11) $K_{11}$ is the set of all multifunctions preserve the predicate

$$
R_{11}=\left(\begin{array}{ccccccccccccccccccc}
0 & 0 & 0 & 1 & 1 & 0 & 0 & - & - & 0 & 1 & - & * & * & * & * & * & * & * \\
0 & 0 & 1 & 0 & 1 & 0 & - & 0 & - & * & * & * & 0 & 1 & - & * & * & * & * \\
0 & 1 & 0 & 0 & 1 & - & 0 & 0 & - & * & * & * & * & * & * & 0 & 1 & - & *
\end{array}\right) .
$$

12) $K_{12}$ is the set of all multifunctions preserve the predicate

$$
R_{12}=\left(\begin{array}{ccccccccccccccccccc}
0 & 0 & 1 & 1 & 1 & 1 & 1 & - & - & 0 & 1 & - & * & * & * & * & * & * & * \\
0 & 1 & 0 & 1 & 1 & 1 & - & 1 & - & * & * & * & 0 & 1 & - & * & * & * & * \\
0 & 1 & 1 & 0 & 1 & - & 1 & 1 & - & * & * & * & * & * & * & 0 & 1 & - & *
\end{array}\right) .
$$

For any multifunction $f$ we uniquely define vector $\tau(f)=\left(\tau_{1}, \ldots, \tau_{12}\right)$. This vector $\tau(f)$ is a vector of membership in the sets $K_{1}-K_{12}$ and for every $i \in\{1, \ldots, 12\} \tau_{i}=\left\{\begin{array}{ll}0 & \text { if } f \in K_{i} \\ 1 & \text { if } f \notin K_{i}\end{array}\right.$.

The membership relation in the sets $K_{1}-K_{12}$ is an equivalence relation and generates a partition of $P_{2}^{*}$ into equivalence classes. For multifunctions from one class, the membership vectors in the sets $K_{1}-K_{12}$ are the same. Since there are 12 maximal partial ultraclones, the largest possible number of equivalence classes is $2^{12}=4096$.

In this paper, we find the number of equivalence classes that consist only of hyperfunctions.

\section{The main result}

In [1] it is shown that the set of Boolean functions is divided into 15 equivalence classes with respect to membership in the maximal partial ultraclones. Therefore, throughout the paper, we consider only hyperfunctions from the set $P_{2}^{-} \backslash P_{2}$. Obviously, any hyperfunction belongs to the $K_{6}$ and does not belong to the $K_{7}$.

Lemma 1. For any $f \in P_{2}^{-} \backslash P_{2}$ the following statements are true:

1) $f \notin K_{5}$

2) if $f$ is not the constant hyperfunction - , then $f \notin K_{10}$,

3) $f \in K_{1} \cap K_{2}$ if and only if $f \in K_{3}$.

Proof. 1) Let $f$ be an arbitrary hyperfunction from the set $P_{2}^{-} \backslash P_{2}$. There must be a tuple $\tilde{\alpha}$ such that $f(\tilde{\alpha})=-$. Moreover, it is obvious that $f(\overline{\tilde{\alpha}}) \in\{0,1,-\}$. Therefore, $f \notin K_{5}$.

2) Let $f$ be an arbitrary hyperfunction other than the constant hyperfunction - . There are tuples $\tilde{\alpha}^{1}$ and $\tilde{\alpha}^{2}$ such that $f\left(\tilde{\alpha}^{1}\right)=-$ and $f\left(\tilde{\alpha}^{2}\right)=\lambda \in\{0,1\}$. Then $f\left(\begin{array}{c}\tilde{\alpha}^{1} \\ \tilde{\alpha}^{1} \\ \tilde{\alpha}^{2} \\ \tilde{\alpha}^{2}\end{array}\right)=\left(\begin{array}{c}- \\ - \\ \lambda \\ \lambda\end{array}\right) \notin R_{10}$, where column $\left(\alpha_{i}^{1} \alpha_{i}^{1} \alpha_{i}^{2} \alpha_{i}^{2}\right)^{t}$ belongs to the predicate $R_{10}$ for any $i \in\{1, \ldots, n\}$.

3) The assertion follows directly from the definitions of the $K_{1}, K_{2}, K_{3}$.

Lemma 2. For any $f \in P_{2}^{-} \backslash P_{2}$ the following statements are true:

1) if $f \notin K_{8}$ then $f \notin K_{11}$,

2) if $f \notin K_{9}$ then $f \notin K_{12}$.

Proof. 1) Let $f \notin K_{8}$. Suppose that $f$ does not satisfy the first condition in the definition of the $K_{8}$. There are tuples $\tilde{\alpha}^{i}=\left(\alpha_{1}^{i}, \ldots, \alpha_{n}^{i}\right)$, where $i \in\{1,2,3\}$, such that column $\left(\alpha_{j}^{1} \alpha_{j}^{2} \alpha_{j}^{3}\right)^{t}$ coincides with one of the columns $(000)^{t},(001)^{t},(010)^{t},(111)^{t}$ for any $j$ and $f\left(\begin{array}{c}\tilde{\alpha}^{1} \\ \tilde{\alpha}^{2} \\ \tilde{\alpha}^{3}\end{array}\right) \in\left\{\left(\begin{array}{l}1 \\ 0 \\ 0\end{array}\right),\left(\begin{array}{l}0 \\ 1 \\ 1\end{array}\right),\left(\begin{array}{l}1 \\ 0 \\ 1\end{array}\right),\left(\begin{array}{l}1 \\ 1 \\ 0\end{array}\right)\right\}$. If $f\left(\begin{array}{c}\tilde{\alpha}^{1} \\ \tilde{\alpha}^{2} \\ \tilde{\alpha}^{3}\end{array}\right) \in\left\{\left(\begin{array}{l}0 \\ 1 \\ 1\end{array}\right),\left(\begin{array}{l}1 \\ 0 \\ 1\end{array}\right),\left(\begin{array}{l}1 \\ 1 \\ 0\end{array}\right)\right\}$, then considering 
that $(000)^{t},(001)^{t},(010)^{t},(111)^{t} \in R_{11}$ and $(011)^{t},(101)^{t},(110)^{t} \notin R_{11}$, we get $f \notin K_{11}$. If $f\left(\begin{array}{c}\tilde{\alpha}^{1} \\ \tilde{\alpha}^{2} \\ \tilde{\alpha}^{3}\end{array}\right)=\left(\begin{array}{l}1 \\ 0 \\ 0\end{array}\right)$, then $f\left(\begin{array}{c}\tilde{\alpha}^{1} \\ \tilde{\beta} \\ \tilde{\alpha}^{3}\end{array}\right)=\left(\begin{array}{c}1 \\ - \\ 0\end{array}\right) \notin R_{11}$, where the tuple $\tilde{\beta}=\left(\beta_{1}, \ldots, \beta_{n}\right)$ is such that $\beta_{k}=-$ for those $k$ for which $\left(\alpha_{k}^{1} \alpha_{k}^{2} \alpha_{k}^{3}\right)^{t}=(010)^{t}$, and $\beta_{k}=\alpha_{k}^{2}$ for the remaining $k$. Therefore, $f \notin K_{11}$.

Now suppose that $f$ does not satisfy the second condition in the definition of the $K_{8}$. There are tuples $\tilde{\alpha}^{1}$ and $\tilde{\alpha}^{2}$ such that $f\left(\tilde{\alpha}^{1}\right)=-$ and $f\left(\tilde{\alpha}^{2}\right)=1$. Consider the value of $f$ on the tuple consisting only of 1 . If $f(\tilde{1}) \in\{0,1\}$, then $f\left(\begin{array}{c}\tilde{\alpha}^{1} \\ \tilde{1} \\ \tilde{\alpha}^{1}\end{array}\right) \in\left\{\left(\begin{array}{c}- \\ 0 \\ -\end{array}\right),\left(\begin{array}{c}- \\ 1 \\ -\end{array}\right)\right\} \notin R_{11}$. If $f(\tilde{1})=-$, then $f\left(\begin{array}{c}\tilde{\alpha}^{2} \\ \tilde{1} \\ \tilde{\alpha}^{2}\end{array}\right)=\left(\begin{array}{c}1 \\ - \\ 1\end{array}\right) \notin R_{11}$. Therefore, $f \notin K_{11}$.

2) The proof is similar to the proof of the preceding item due to duality.

Lemma 3. For any $f \in P_{2}^{-} \backslash P_{2}$ the following statements are true:

1) if $f \in K_{1}$, then $f \notin K_{9}$ and $f \notin K_{12}$;

2) if $f \in K_{2}$, then $f \notin K_{8}$ and $f \notin K_{11}$;

3) if $f \in K_{1} \bigcap K_{2}$, then $f \notin K_{8} \bigcup K_{9}$ and $f \notin K_{11} \bigcup K_{12}$.

Proof. 1) Let $f \in K_{1}$. Then $f(\tilde{0})=0$, i.e. there is the tuple on which the value of $f$ is equal to 0 . So, taking into account the mandatory existence of a tuple on which the value of the function $f$ is equal to -, we obtain that the hyperfunction $f$ does not satisfy the second condition in the definition of the $K_{9}$. Therefore, $f \notin K_{9}$ and, by the point 2) of Lemma 2, we obtain that $f \notin K_{12}$.

2) The proof is similar to the proof of the preceding item due to duality.

3) The validity of the statement follows from the items 1) and 2) of the present Lemma, as well as the items 1) and 2) of Lemma 2.

Lemma 4. Let $f \in P_{2}^{-} \backslash P_{2}$. If $f \in K_{1} \backslash K_{2}$ or $f \in K_{2} \backslash K_{1}$, then $f \notin K_{4}$.

Proof. For definiteness, let $f \in K_{1} \backslash K_{2}$. Then $f(\tilde{0})=0$ and $f(\tilde{1}) \in\{0,-\}$. We show that in each case the hyperfunction $f$ does not satisfy the conditions in the definition of the $K_{4}$. If $f(\tilde{1})=0$, then $f(\overline{\tilde{1}})=f(\tilde{0})=0 \neq 1=\overline{f(\tilde{0})}=\overline{f(\overline{\tilde{1}})}$. If $f(\tilde{1})=-$, then $f(\overline{\tilde{1}})=f(\tilde{0})=0 \neq-$. In the case when the hyperfunction $f$ belongs to the set $K_{2} \backslash K_{1}$, the proof is similar.

Lemma 5. For any $f \in P_{2}^{-} \backslash P_{2}$ the following statements are true:

1) if $f \in K_{8} \cap K_{9}$, then $f$ is the constant hyperfunction -;

2) if $f \in K_{11} \cap K_{12}$, then $f$ is the constant hyperfunction-.

Proof. 1) Suppose $f$ is not the constant hyperfunction -. Then there is a tuple $\tilde{\alpha}$ such that $f(\tilde{\alpha})=\lambda \in\{0,1\}$. There is necessarily a tuple in which the value of $f$ is equal to - . So, if $\lambda=0$, then $f$ does not satisfy the second condition in the definition of the $K_{9}$, if $\lambda=1$, then $f$ does not satisfy the second condition in the definition of the $K_{8}$. Therefore, either $f \notin K_{9}$ or $f \notin K_{8}$, which contradicts the fact that $f$ belongs to the set $K_{8} \bigcap K_{9}$.

2) Suppose $f$ is not the constant hyperfunction -. From the previous item, we obtain either $f \notin K_{9}$ or $f \notin K_{8}$. Further, using the assertions of Lemma 2, we obtain that either $f \notin K_{12}$ or $f \notin K_{11}$, which contradicts the fact that $f$ belongs to the set $K_{11} \bigcap K_{12}$.

Lemma 6. Let $f \in P_{2}^{-} \backslash P_{2}$. If $f \notin K_{1} \bigcup K_{2}$ and $f \in K_{4}$, then either $f$ is the constant hyperfunction - or $f \notin K_{8} \bigcup K_{9}$ and $f \notin K_{11} \bigcup K_{12}$. 
Proof. Since $f \notin K_{1} \cup K_{2}$, then $f(\tilde{0}) \in\{1,-\}$ and $f(\tilde{1}) \in\{0,-\}$. Considering that $f \in K_{4}$ it is enough to consider cases $f(\tilde{0})=1, f(\tilde{1})=0$ and $f(\tilde{0})=f(\tilde{1})=-$. In the case when $f(\tilde{0})=1$, $f(\tilde{1})=0$ as well as in the proof of Lemma 3, we get that $f \notin K_{8} \bigcup K_{9}$ and $f \notin K_{11} \bigcup K_{12}$. If $f(\tilde{0})=f(\tilde{1})=-$, then either $f$ is the constant hyperfunction - and the statement of the Lemma holds, or there is a tuple $\tilde{\alpha}$ such that $f(\tilde{\alpha}) \in\{0,1\}$. Without loss of generality, we can assume that $f(\tilde{\alpha})=0$. Since $f \in K_{4}$, then $f(\overline{\tilde{\alpha}})=1$. Thus, there are tuples on which the hyperfunction $f$ is equal to 0,1 , and - . Therefore, $f \notin K_{8} \bigcup K_{9}$ and $f \notin K_{11} \bigcup K_{12}$.

Lemma 7. Let $f \in P_{2}^{-} \backslash P_{2}$. If $f \notin K_{1} \cup K_{2}$ and $f \notin K_{4}$, then $f \notin K_{11} \bigcup K_{12}$.

Proof. Since $f \notin K_{1} \cup K_{2}$, then $f(\tilde{0}) \in\{1,-\}$ and $f(\tilde{1}) \in\{0,-\}$. For the cases when $f(\tilde{0})$ and $f(\tilde{1})$ are not equal to - simultaneously, we have $f\left(\begin{array}{c}\tilde{0} \\ \tilde{1} \\ \tilde{0}\end{array}\right) \in\left\{\left(\begin{array}{l}1 \\ 0 \\ 1\end{array}\right),\left(\begin{array}{c}1 \\ - \\ 1\end{array}\right),\left(\begin{array}{c}- \\ 0 \\ -\end{array}\right)\right\} \notin R_{11}$ and $f\left(\begin{array}{c}\tilde{1} \\ \tilde{0} \\ \tilde{1}\end{array}\right) \in\left\{\left(\begin{array}{l}0 \\ 1 \\ 0\end{array}\right),\left(\begin{array}{c}0 \\ - \\ 0\end{array}\right),\left(\begin{array}{c}- \\ 1 \\ -\end{array}\right)\right\} \notin R_{12}$. Suppose $f(\tilde{0})=f(\tilde{1})=-$. Since $f \notin K_{4}$, it is not the constant hyperfunction - and, so, there is a tuple $\tilde{\alpha}$ such that $f(\tilde{\alpha})=\lambda \in\{0,1\}$. Then $f\left(\begin{array}{c}\tilde{0} \\ \tilde{\alpha} \\ \tilde{0}\end{array}\right)=\left(\begin{array}{c}- \\ \lambda \\ -\end{array}\right) \notin R_{11}$ and $f\left(\begin{array}{c}\tilde{1} \\ \tilde{\alpha} \\ \tilde{1}\end{array}\right)=\left(\begin{array}{c}- \\ \lambda \\ -\end{array}\right) \notin R_{12}$.

Theorem 1.1. The set of all hyperfunctions of rank 2 other than Boolean functions generates no more than 13 equivalence classes with respect to membership in the maximal partial ultraclones.

Proof. From the first two points of Lemma 1, it follows that for any of the considered hyperfunctions $f$ the components $\tau_{5}$ and $\tau_{10}$ of the vector $\tau(f)=\left(\tau_{1} \tau_{2} \tau_{3} \tau_{4} \tau_{5} 01 \tau_{8} \tau_{9} \tau_{10} \tau_{11} \tau_{12}\right)$ are equal to 1 , where $\tau(f)$ is the vector of membership in the $K_{1}-K_{12}$. From the third point of the Lemma 1 we get that $\left(\tau_{1} \tau_{2} \tau_{3}\right) \in\{(000),(011),(101),(111)\}$. Consider all these cases.

From the third point of Lemma 3, it follows that the hyperfunctions belonging simultaneously to the $K_{1}, K_{2}, K_{3}$ are divided into no more than 2 equivalence classes, these classes correspond to the vectors (000010111111), (000110111111).

Now consider the hyperfunctions that either belong to $K_{1}$ and do not belong to $K_{2}, K_{3}$, or belong to $K_{2}$ and do not belong to $K_{1}, K_{3}$. Using Lemma 2, the first two points of Lemma 3 and Lemma 4, we obtain that the number of equivalence classes for such hyperfunction is no more than 6 and the vectors corresponding to these classes have the form (011110101101), (011110101111), (011110111111), (101110110110), (101110110111), (101110111111).

It remains to consider hyperfunctions that do not belong to any of the $K_{1}, K_{2}, K_{3}$. It is obvious that among such hyperfunctions there are those that take the value - on each tuple. It is easy to verify that the vector of membership in the $K_{1}-K_{12}$ for these hyperfunctions has the form (111010100000). Further we assume that hyperfunctions are not constant. By Lemma 6 , we obtain that hyperfunctions belonging to the $K_{4}$ can generate at most one equivalence class, to which the membership vector (111010111111) corresponds. Further, applying Lemmas 5 and 7 , we obtain that hyperfunctions that do not belong to the $K_{4}$ are divided into no more than 3 equivalence classes, which correspond to the vectors (111110101111), (111110110111), (111110111111).

Theorem 1.2. The set of all hyperfunctions of rank 2 generates 28 equivalence classes with respect to membership in the maximal partial ultraclones.

Proof. Since the number of classes of Boolean functions is 15 considering the previous theorem, we obtain that all hyperfunctions are divided into no more than 28 equivalence classes. 
As a result of computer calculations on hyperfunctions of three variables, 28 different vectors of membership in the $K_{1}-K_{12}$ were found. The Tab. 1 shows the vectors of affiliation and the corresponding hyperfunctions. Table 1 shows membership vectors and the corresponding hyperfunctions. Note that at number 23 there is the constant hyperfunction -.

Table 1

\begin{tabular}{|c|c|c||c|c|c|}
\hline $\mathrm{N}$ & $\tau(f)$ & $f\left(x_{1}, x_{2}, x_{3}\right)$ & № & $\tau(f)$ & $f\left(x_{1}, x_{2}, x_{3}\right)$ \\
\hline 1 & $(000000000000)$ & $(00001111)$ & 15 & $(101110000000)$ & $(1111111)$ \\
2 & $(000000011011)$ & $(01101001)$ & 16 & $(101110011011)$ & $(10011001)$ \\
3 & $(000000011111)$ & $(00010111)$ & 17 & $(101110011111)$ & $(10000001)$ \\
4 & $(000010111111)$ & $(000--111)$ & 18 & $(101110110110)$ & $(-1111111)$ \\
5 & $(000110001101)$ & $(00000001)$ & 19 & $(101110110111)$ & $(111111-1)$ \\
6 & $(000110010110)$ & $(00111111)$ & 20 & $(101110111111)$ & $(100000-1)$ \\
7 & $(000110011111)$ & $(00000111)$ & 21 & $(111000011011)$ & $(10010110)$ \\
8 & $(000110111111)$ & $(000000-1)$ & 22 & $(111000011111)$ & $(10001110)$ \\
9 & $(011110000000)$ & $(00000000)$ & 23 & $(111010100000)$ & - \\
10 & $(011110011011)$ & $(00111100)$ & 24 & $(111010111111)$ & $(100--110)$ \\
11 & $(011110011111)$ & $(00000010)$ & 25 & $(111110011111)$ & $(10000000)$ \\
12 & $(011110101101)$ & $(0000000-)$ & 26 & $(111110101111)$ & $(-0000000)$ \\
13 & $(011110101111)$ & $(000000-0)$ & 27 & $(111110110111)$ & $(1111111-)$ \\
14 & $(011110111111)$ & $(0000001-)$ & 28 & $(111110111111)$ & $(1000000-)$ \\
\hline
\end{tabular}

This work was supported by RFBR according to the research project no. 18-31-00020.

\section{References}

[1] S.A.Badmaev, On the Classes of Boolean Functions Generated by Maximal Partial Ultraclones, The Bulletin of Irkutsk State University. Series Mathematics, 27(2019), 3-14 (in Russian).

[2] S.V.Zamaratskaya, V.I.Panteleev, Classification and Types of Bases of All Ultrafunctions on Two-Element Set, The Bulletin of Irkutsk State University. Series Mathematics, 16(2016), 58-70 (in Russian).

[3] A.S.Zinchenko, V.I.Panteleev, On Classes of Hyperfunctions of Rank 2 Generated by Maximal Multiclones, The Bulletin of Irkutsk State University. Series Mathematics, 21(2017), 61-76 (in Russian).

[4] A.S.Kazimirov, V.I. Panteleyev, L.V.Tokareva, Classification and Enumeration of Bases in Clone of All Hyperfunctions on Two-Elements Set, The Bulletin of Irkutsk State University. Series Mathematics, 7(2014), 61-78 (in Russian).

[5] A.S.Kazimirov, V.I.Panteleyev, On the Classes of Boolean Functions Generated by Maximal Multiclones, The Bulletin of Buryat State University. Mathematics and Informatics, 9(2015), 16-22 (in Russian).

[6] S.V.Yablonskij, On the Superpositions of Logic Functions, Mat. Sbornik, bf 30(1952), no. 2(72), 329-348 (in Russian). 
[7] M.Miyakawa, I.Stojmenović, D.Lau, I.Rosenberg, Classification and Basis Enumerations in Many-Valued Logics, Proc. 17th International Symposium on Multi-Valued logic. Boston, 1987, 151-160.

[8] M.Miyakawa, I.Stojmenović, D.Lau, I.Rosenberg, Classification and Basis Enumerations of the Algebras for Partial Functions, Proc. 19th International Symposium on Multi-Valued logic. Rostock, 1989, 8-13.

[9] S.A.Badmaev, On Some Maximal Clone of Partial Ultrafunctions on a Two- Element Set, Journal of Siberian Federal University. Mathematics and Physics, 10(2017), no. 2, 140-145.

[10] S.A.Badmaev, A Completeness Criterion of Set of Multifunctions in Full Partial Ultraclone of Rank 2, Siberian Electronic Mathematical Reports, 15(2018), 450-474 (in Russian).

\section{Классификация гиперфункций ранга 2 относительно принадлежности максимальным частичным ультраклонам}

Сергей А. Бадмаев

Институт математики и информатики Бурятский государственный университет

Смолина, 24а, Улан-Удэ, 670000

Россия

$\overline{\text { В данной работе рассматривается множество гиперфункиий, которое является подмножеством }}$ полного частичного ультраклона ранга 2. Для гиперфункций решена задача их классификации относительно принадлежности максимальным частичным ультраклонам. Отношение принадлежности максимальным частичным ультраклонам является отношением эквивалентности и порождает соответствующее разбиение на классы эквивалентности. Получено полное описание всех классов эквивалентности, общее число которых равно 28.

Ключевые слова: мультифункиия, гиперфункиия, клон, ультраклон, максимальный частичный ультраклон, классификачия функций. 